\title{
Intense terahertz radiation from relativistic laser-plasma interactions
}

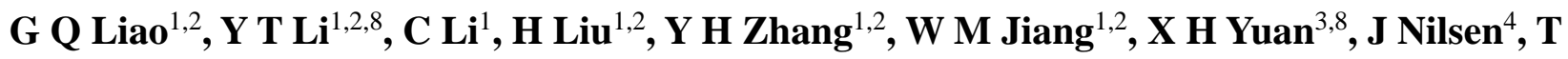

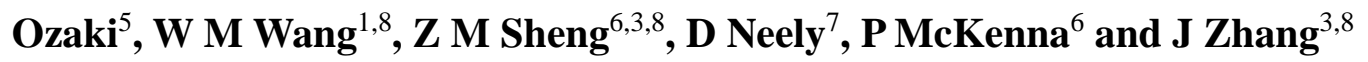 \\ ${ }^{1}$ Beijing National Laboratory for Condensed Matter Physics, Institute of Physics, Chinese Academy of \\ Sciences, Beijing 100190, China \\ ${ }^{2}$ School of Physical Sciences, University of Chinese Academy of Sciences, Beijing 100190, China \\ ${ }^{3}$ Key Laboratory for Laser Plasmas (MoE) and Department of Physics and Astronomy, Shanghai Jiao Tong \\ University, Shanghai 200240, China \\ ${ }^{4}$ Lawrence Livermore National Laboratory, 7000 East Avenue, Livermore, CA 94551, USA \\ ${ }^{5}$ INRS-EMT, 1650 Boulevard Lionel-Boulet, Varennes, Québec J3X 1S2, Canada \\ ${ }^{6}$ SUPA, Department of Physics, University of Strathclyde, Glasgow G4 0NG, United Kingdom \\ ${ }^{7}$ Central Laser Facility, STFC Rutherford Appleton Laboratory, Didcot, Oxfordshire OX11 0QX, United \\ Kingdom \\ ${ }^{8}$ Collaborative Innovation Center of IFSA (CICIFSA), Shanghai Jiao Tong University, Shanghai 200240, \\ China
}

E-mail: ytli@iphy.ac.cn

\begin{abstract}
The development of tabletop intense terahertz $(\mathrm{THz})$ radiation sources is significantly important for THz science and applications. This paper presents our measurements of intense $\mathrm{THz}$ radiation from relativistic laser-plasma interactions under different experimental conditions. Several THz generation mechanisms have been proposed and investigated, including coherent transition radiation emitted by fast electrons from the target rear surface, transient current radiation at the front of the target, and mode conversion from electron plasma waves to $\mathrm{THz}$ waves. The results indicate that relativistic laser plasma is a promising driver of intense $\mathrm{THz}$ radiation sources.
\end{abstract}

Keywords: intense terahertz radiation, laser-plasma interactions, coherent transition radiation, transient current, mode conversion 


\section{Introduction}

Terahertz $(\mathrm{THz})$ radiation has attracted much interest for its promising applications in many fields such as material [1], bio-medical [2], and communication [3], etc. During the last decades ultrafast intense lasers have been used to drive $\mathrm{THz}$ radiation $[4,5,6,7,8]$. Among them plasma-based $\mathrm{THz}$ sources have been paid much attention since plasma is free of optical damages [9]. THz radiation from laser-induced plasma filaments in air or in other low-density gases has been investigated extensively $[10,11,12]$. However, due to the ionization-induced laser defocusing in the filaments, the $\mathrm{THz}$ yield is found to be saturated with the pump laser intensity higher than $10^{15} \mathrm{~W} / \mathrm{cm}^{2}[13]$.

Currently the focused intensity of ultraintense laser pulses can be well above $10^{18} \mathrm{~W} / \mathrm{cm}^{2}$. To take the advantages of such a high laser intensity, a few groups have investigated the generation of stronger $\mathrm{THz}$ radiation in relativistic laser-plasma interactions. For example, Leemans et al. obtained $\mathrm{THz}$ radiation with energies of sub- $\mu \mathrm{J}$ from intense laser-gas interactions [14]. Hamster et al. found that the $\mathrm{THz}$ radiation generated from solid targets was three orders of magnitude stronger than that from gas targets [15]. Sagisaka et al. [16] and Gao et al. [17] discussed the THz generation with an antenna model. Similarly, Tokita et al. [18] and Poyé et al. [19] investigated the THz radiation generated by the transient charge separation along the target [20]. Recently Gopal et al. obtained very strong $\mathrm{THz}$ radiation with energies of $\sim 700 \mu \mathrm{J}$ from the rear of a foil target [21], which was attributed to the target normal sheath acceleration (TNSA) [22].

The generation mechanisms of $\mathrm{THz}$ radiation from laser-solid interactions are complicated. To clarify them, we have systematically investigated the $\mathrm{THz}$ radiation from either the front or the rear of solid targets irradiated by relativistic femtosecond and picosecond laser pulses. With experimental observations and particle-in-cell (PIC) simulations, we have proposed and demonstrated three scenarios for different laser plasma conditions, which are highlighted in this paper.

\section{Coherent transition radiation in the $\mathrm{THz}$ regime}

High charge ( $\mathrm{nC}-\mu \mathrm{C}) \mathrm{MeV}$ fast electrons can be generated during intense laser-solid interactions. For a foil target, fast electrons transport forward through the target and will induce transition radiation 
when crossing the rear surface-vacuum boundary. Usually the bunch length of the fast electrons accelerated by a laser pulse in tens of femtosecond duration is of the order of $\sim 10 \mu \mathrm{m}$, which is smaller than the wavelength of $\mathrm{THz}$ radiation. This will lead to the coherent transition radiation (CTR), in which the total radiation energy scales with the square of the electron number [23]. One can expect that the THz radiation energy will be high due to the high charge and short bunch duration of the fast electron beam as well as the steep foil-vacuum boundary.

To verify this idea, we have carried out a relativistic laser-foil interaction experiment using the femtosecond laser system at the Laboratory for Laser Plasma, Shanghai Jiao Tong University [24]. A p-polarized laser pulse in $30 \mathrm{fs}$ and $2 \mathrm{~J}$ was incident onto solid targets at an incidence angle of $54^{\circ}$ with a peak intensity of $\sim 1.5 \times 10^{19} \mathrm{~W} / \mathrm{cm}^{2}$. The laser prepulse contrast in the ns range is $\sim 10^{-5}$. The $\mathrm{THz}$ radiation was collected at $-75^{\circ}$ with respect to the target rear normal, and measured by a pyroelectric detector. A set of low-pass or narrowband band-pass filters was used to measure the radiation spectra. Different types of targets were used in the experiment, including mass-limited metal targets with different sizes, polyethylene (PE)-metal double-layered, single PE and $\mathrm{Cu}$ targets.

Figure 1(a) shows the frequency spectrum of $\mathrm{THz}$ radiation measured with $5 \mu \mathrm{m}$ thick $\mathrm{Cu}$ foils. The radiation covers a bandwidth up to $30 \mathrm{THz}$. The theory of CTR predicts the radiation wavelength $\lambda$ to be larger than $\sigma_{\mathrm{ez}}[25]$, where $\sigma_{\mathrm{ez}}$ is the longitudinal length of electron bunches. In the present case, the bunch length of laser ponderomotive accelerated electrons is estimated to be $\sigma_{\mathrm{ez}} \sim c \cdot \tau_{\mathrm{L}} \sim 10$ $\mu \mathrm{m}$, where $c$ is the velocity of light and $\tau_{\mathrm{L}}$ is the laser pulse duration. Therefore, the generated coherent radiation is estimated to be mainly within $30 \mathrm{THz}$, which agrees with the measurement. If the laser contrast was high enough, electrons would be mainly accelerated through vacuum heating with characteristic micro-bunching structures separated by the laser period [26]. Nevertheless, as long as the micro-bunches are shorter than the THz radiation wavelength, the transition radiation emitted by the micro-bunched electrons will remain coherent in the $\mathrm{THz}$ regime. 

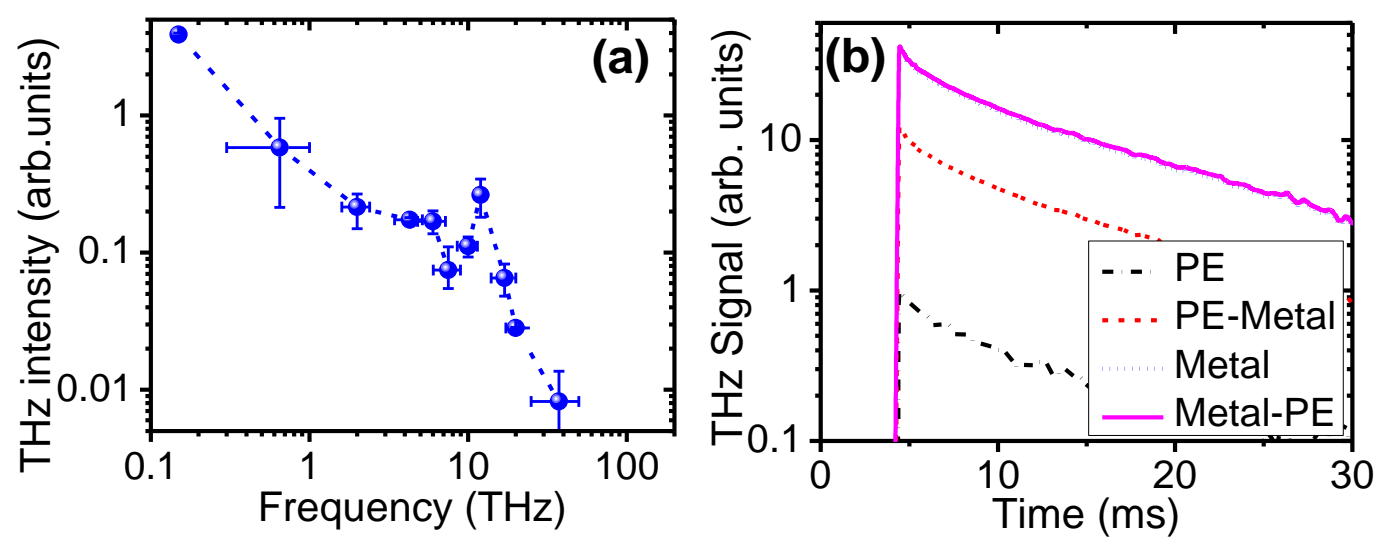

Figure 1. (a) Experimentally measured $\mathrm{THz}$ radiation spectrum at $-75^{\circ}$ from the rear side of $5 \mu \mathrm{m}$ thick metal foils (From Liao et al 2016 [24]). (b) Comparison of the typical THz signals measured with different targets.

Transition radiation depends critically on the dielectric property of medium boundary. Figure 1(b) compares the typical THz signals measured with different targets, where the PE layer is $40 \mu \mathrm{m}$ thick and the metal layer $5 \mu \mathrm{m}$ thick, respectively. The laser pulse was focused on the front PE surface for the PE/PE-metal targets or the metal surface for the metal/metal-PE targets. The peak intensity ratio of THz radiation from different targets is PE: PE-metal: Metal: Metal-PE=1: 12: 39: 41. When a $5 \mu \mathrm{m}$ thick metal layer is coated behind the PE layer, the THz radiation is dramatically enhanced by over 10 times. The relative dielectric constant of the $\mathrm{PE}$ at the $\mathrm{THz}$ regime is $\sim 2.3$, while that of the metal is much greater than 1 [27]. Stronger THz radiation with a metallic rear layer is a direct evidence for transition radiation. Moreover, the $\mathrm{THz}$ radiation from the metal and metal-PE targets is stronger than those from the PE and PE-metal targets. This is due to that the metal surface irradiated by laser pulses will lead to a higher laser absorption efficiency, fast electron number and electron temperature than the PE surface $[28,29]$. The slightly stronger $\mathrm{THz}$ radiation from the metal-PE targets than the single-layer metal targets could result from the two CTR sources at the metal-PE and PE-vacuum interfaces for the metal-PE targets [24].

Figure 2 shows the dependence of $\mathrm{THz}$ radiation from mass-limited targets on the target size. The THz intensity is increased dramatically when the target size increases from $200 \times 200 \mu \mathrm{m}^{2}$ to $1 \times 1$ $\mathrm{mm}^{2}$, and then seems saturated as the target size increases from $1 \times 1 \mathrm{~mm}^{2}$ to $2 \times 2 \mathrm{~mm}^{2}$. This can be explained by the CTR model modified by diffraction radiation. Compared with the transition radiation from an infinite interface, the radiation from targets of a finite size will be reduced by the diffraction modification factor, $D$ [25]. The curves in figure 2 show the theoretically calculated $D$ as a function 
of target sizes with different relativistic factors of fast electrons $\gamma$ and radiation wavelength $\lambda$. One can see the calculated curve with $\lambda=300 \mu \mathrm{m}$ gives the closest agreement to the experimental results.

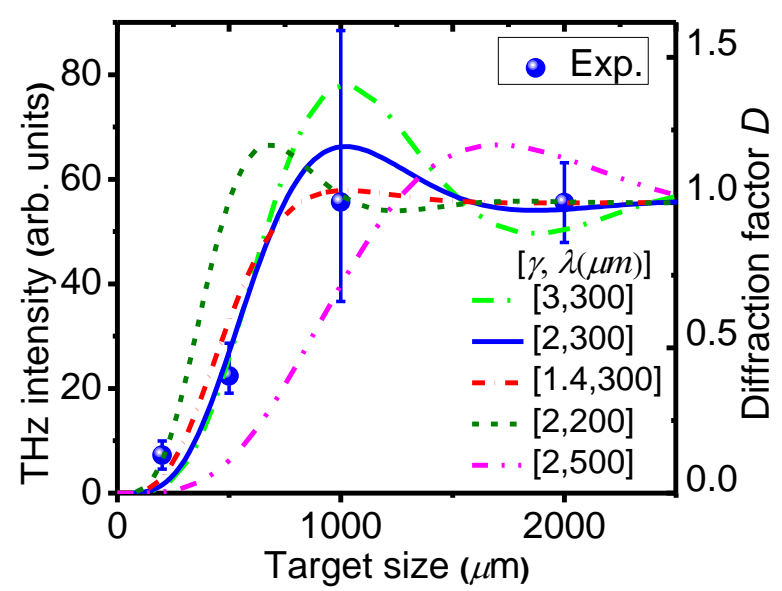

Figure 2. Experimentally measured $\mathrm{THz}$ intensity at $-75^{\circ}$ (blue circles) and theoretically calculated diffraction modification factor $D$ (curves) as a function of target sizes. (Reproduced with permission from [24]. Copyright 2016, American Physical Society.)

When the fast electrons cross the target rear surface, a strong sheath field will be established due to the transient charge separation, leading to the well-known TNSA. The time-varying dipole-like charge structure formed during the TNSA has been proposed to emit $\mathrm{THz}$ radiation as an antenna [22]. According to this model, the THz radiation should be correlated with the ion acceleration. To check the CTR or the TNSA dipole-like radiation is dominant in our experiment, we have measured both the $\mathrm{THz}$ radiation and the ion acceleration with mass-limited targets. We find that both the number and maximum energy of ions are enhanced significantly with the decrease of target sizes [24]. The different dependence of the $\mathrm{THz}$ radiation and ion acceleration on target sizes indicates that the TNSA dipole-like radiation is not the dominant $\mathrm{THz}$ generation mechanism under our experimental conditions.

The sheath field could ionize the target rear, forming a thin plasma layer. Nevertheless, according to the model of CTR [30], as long as the plasma scalelength at the target rear is much less than the formation length of CTR (in the order of $\sim 100 \mu \mathrm{m}$ for THz radiation), the rear plasma layer could still be approximately considered as a sharp boundary. Since the typical scale of the rear plasma is $\sim$ several micrometers in a ps timescale during which the fast electrons cross the target, the overall 
CTR process in the THz regime will not be affected much by the rear plasma. On the other hand, the sheath field at the target rear could pull some fast electrons back within a few $\mu$ m distance. According to the theory of CTR, fast electrons have to propagate over a formation length to efficiently deliver the energy to electromagnetic radiation [31]. Hence, only those forward electrons escaping from the target surface (not those pulled back by the sheath field) contribute to the CTR observed.

According to the experimental observation and the theoretical model of CTR, the total $\mathrm{THz}$ energy from the rear of metal foils is estimated to be $\sim 400 \mu \mathrm{J} /$ pulse, comparable to the energy level of the conventional accelerator based $\mathrm{THz}$ sources [32]. The corresponding energy conversion efficiency from the laser pulse energy on targets to $\mathrm{THz}$ radiation is $\sim 2 \times 10^{-4}$.

\section{Target surface transient current}

Fast electrons generated in laser-solid interactions transport not only longitudinally but also laterally, forming strong transient currents along the target surface. The time-varying transient current in the low-density plasma region will emit electromagnetic radiation outward. The radiation spectrum mainly depends on the temporal evolution of transient currents. The fast electron current driven by femtosecond laser pulses usually has a timescale in the range from tens of femtoseconds to picosecond, and hence the radiation falls in the THz regime.

Two-dimensional PIC simulations show that there usually exists two kinds of lateral transient currents with different characteristics near the target surface [33]. One is formed by the directional surface fast electron (SFE) beam due to the confinement of the quasistatic magnetic and electric fields (Current I) [34]. A steep plasma density gradient at the target surface and a large incidence angle are beneficial to the Current I. The other is the radial surface current formed by the lateral electron transport in low-density plasmas (Current II). A moderate plasma density scalelength and a small incidence angle are useful to enhance the Current II [33]. In the PIC simulations, strong lateral return currents are formed by "cold" background electrons which almost cancel out the fast electron currents in the high-density region. While in the low-density region, there exists a strong net, transient current without enough return currents. Given that the observable $\mathrm{THz}$ radiation is mainly generated from the low-density plasma region and the $\mathrm{THz}$ field generated in the high-density region cannot be 
emitted out efficiently into vacuum, the effect of return currents is not significant for the THz radiation in the low-density region.

Figure 3(a) shows the simulated temporal evolution of the energy spectra of the electrons emitted into vacuum within $20^{\circ}$ with respect to the target surface (corresponding to the Current I formed by the SFE beam). Due to the effect of the surface quasistatic electric and magnetic fields, electrons at the surface would firstly be pulled in and reflected out of the target, drifting along the target surface. Then some electrons will be accelerated gradually and generate the THz radiation possibly during the prolonged push-reflection (oscillation) processes [35]. A part of those fast electrons with enough energies will escape from the target, forming the SFE beam observed in vacuum [34]. One can see the energy spectra of electrons in the SFE beam are still not stable at 1 ps under the present simulation parameters. The time-varying current lasts $>1 \mathrm{ps}$, and the generated radiation will be with a pulse duration (FWHM) of about $>0.5$ ps and frequencies within 2 THz. Figure 3(b) shows the simulated temporal evolution of the energy spectra of electrons transporting laterally in underdense (density $<10^{19} \mathrm{~cm}^{-3}$ ) plasmas (corresponding to the Current II). This part of relatively low-energy electrons is mainly accelerated by the laser transverse ponderomotive force in our present case. Seen from figure 3(b), the electrons are accelerated immediately just when the laser is incident onto the target, and the acceleration is stopped when the laser pulse is reflected off the target at $\sim 0.2 \mathrm{ps}$. Correspondingly the energy spectra of this part of electrons tend to be stable after $\sim 0.2 \mathrm{ps}$, generating the radiation with a pulse duration (FWHM) of $\sim 0.1 \mathrm{ps}$ and frequencies of $\sim 10 \mathrm{THz}$. Given the fact that the dimension of the lateral transport of fast electron currents is usually comparable to or even larger than the $\mathrm{THz}$ wavelength [36], the first-order electric-dipole radiation approximation is no longer valid [37]. Hence there will still be high-frequency THz radiation emitted despite that the Current II could be roughly symmetric spatially. This has been verified by the PIC simulations [38]. 


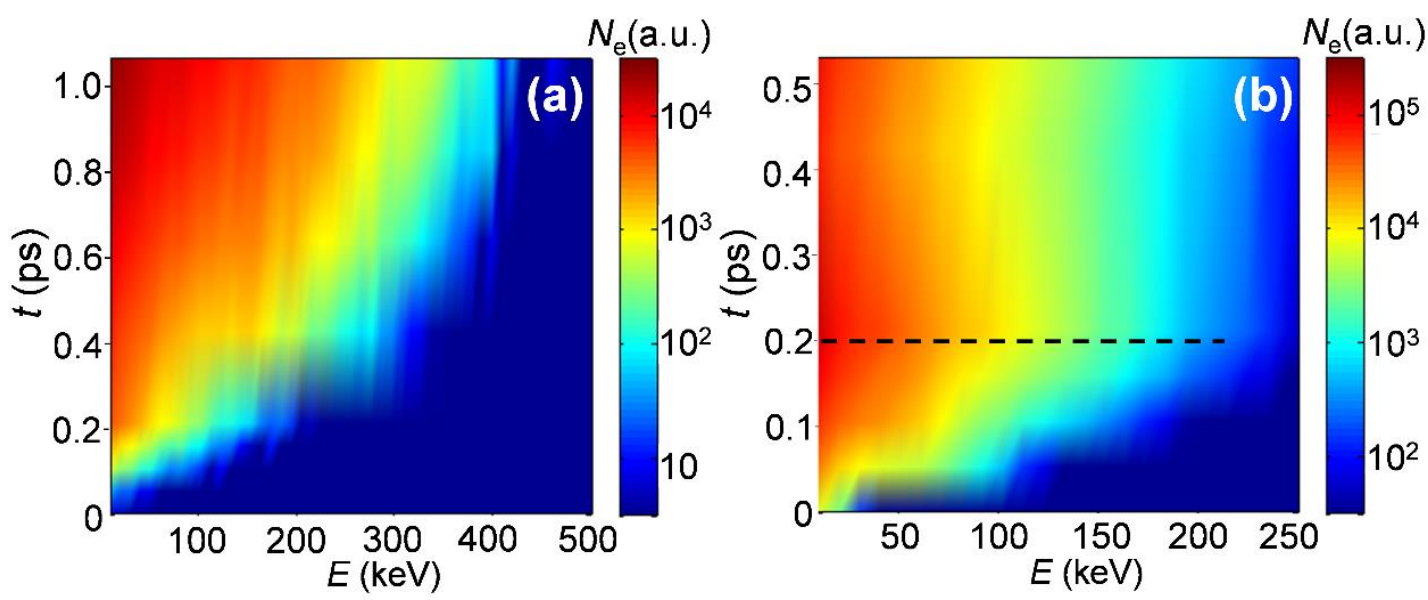

Figure 3. Temporal evolution of the energy spectra of (a) electrons emitted into vacuum within $20^{\circ}$ with respect to the target surface and (b) electrons in the underdense plasma region obtained from PIC simulations. In the simulations, a laser pulse with a duration of $80 \mathrm{fs}$ is incident onto a plasma slab at an incidence angle of $60^{\circ}$. The plasma density increases from $10^{18} \mathrm{~cm}^{-3}$ to $5 \times 10^{21} \mathrm{~cm}^{-3}$ with a density scalelength of $0.1 \mu \mathrm{m}$. From Li et al 2016 [33].

To demonstrate the THz radiation generated by the two kinds of target surface transient currents, we have performed a series of experiments [38,39,40,41] using different laser systems. Typically, an $800 \mathrm{~nm}, 50-100 \mathrm{fs}$ laser pulse was incident onto a $1 \mathrm{~mm}$ thick copper slab at an incidence angle of $67.5^{\circ}, 22.5^{\circ}$ or $10^{\circ}$. The $\mathrm{THz}$ radiation was observed in different directions. The laser pulse energy, contrast and polarization were adjustable in the experiment. A set of THz low-pass filters and a wiregrid $\mathrm{THz}$ polarizer were used to characterize the frequency spectra and polarization of $\mathrm{THz}$ radiation, respectively.

Figure 4(a) and 4(b) shows the THz radiation detected at different observation angles (defined as the angle with respect to the front target normal) $\alpha$ and laser incidence angles $\theta$ as a function of $\mathrm{THz}$ frequency and laser contrast ratio, respectively. The $\mathrm{THz}$ radiation property is found to be closely dependent on $\alpha$ rather than $\theta$. The THz radiation near the target surface $\left(\alpha \geq 67.5^{\circ}\right)$ is dominated by low-frequency $(<3 \mathrm{THz})$ components regardless of the laser incidence angles, as shown with the blue lines in figure $4(\mathrm{a})$. The THz radiation at $\alpha=67.5^{\circ}$ is nearly doubled when $\theta$ is varied from $22.5^{\circ}$ to $67.5^{\circ}$. Seen from figure $4(\mathrm{~b})$, the THz radiation at $\alpha=67.5^{\circ}$ is enhanced significantly by improving the laser contrast. Besides, it is found to be always mainly p-polarized regardless of the laser polarization. Interestingly, the THz radiation near the target normal $\left(\alpha \leq 22.5^{\circ}\right)$ shows much different 
properties from the $\mathrm{THz}$ radiation at $\alpha \geq 67.5^{\circ}$. As shown by the red lines in figure 4 , the $\mathrm{THz}$ radiation at $\alpha=22.5^{\circ}$ is dominated by high-frequency ( $>10 \mathrm{THz}$ ) components, and it is increased with the decrease of laser incidence angles or the increase of preplasma scalelengths. In addition, the polarization orientation of the $\mathrm{THz}$ radiation at $\alpha=22.5^{\circ}$ is found to be roughly the same with the laser polarization, i.e., the $\mathrm{THz}$ radiation becomes mainly s-polarized when the pump laser is $s$ polarized.
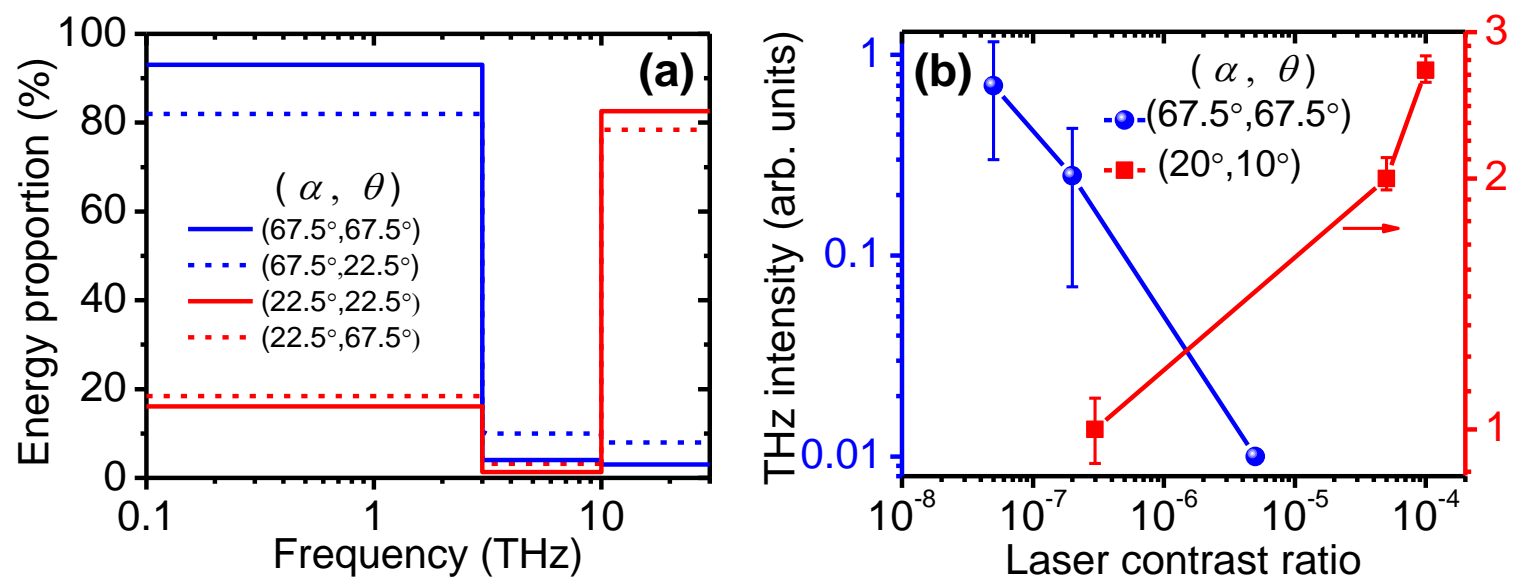

Figure 4. Under different observation angles $\alpha$ and laser incidence angles $\theta$, plot (a) shows the THz energy proportion in different frequency bands and plot (b) shows the $\mathrm{THz}$ intensity as a function of the laser contrast ratio in the ns range.

The dependence of the $\mathrm{THz}$ radiation observed in different directions on the laser-plasma parameters can be explained by the two kinds of target surface transient currents. The low-frequency THz radiation near the target surface is mainly generated by the SFE current (Current I), while the high-frequency one near the target normal by the lateral transport electron current (Current II). In experiments we have also measured the spectra of scattered laser light. The THz radiation near the target surface is found to be correlated with the second harmonics of the laser pulse [40,42], while the radiation near the target normal shows positive correlation with three-halves harmonics [38]. Usually the second and three-halves harmonics are considered as the indication of resonance absorption and two-plasmon-decay instability, respectively. This suggests that the surface transient current could be driven by the two-plasmon-decay instability when there is a moderate preplasma [38]. 


\section{Mode conversion from electron plasma waves to $\mathrm{THz}$ radiation}

In the above surface current model the preplasma scalelength is relatively small. In contrast, when there is a large-scale preplasma presented in front of a solid target, electron plasma waves (EPWs) will be excited during the laser propagation in the plasma. It is well known that a laser electromagnetic wave can be converted into an electrostatic wave at the critical density through resonant absorption [43]. Early studies predicted that there is a reversal symmetry in the electromagnetic-electrostatic mode conversion [44], which means plasma waves can be converted into electromagnetic radiation in certain conditions. Since the amplitude of the converted electromagnetic field is proportional to that of the plasma wave, it is also known as linear mode conversion (LMC). Sheng et al. have proposed that laser wakefields can emit intense $\mathrm{THz}$ radiation around the plasma frequency mainly in the specular direction through LMC when the laser pulse is incident obliquely to an inhomogeneous plasma of a positive density gradient [45]. For example, at the plasma density of $10^{18} \mathrm{~cm}^{-3}$, the frequency of mode-converted radiation lies $\sim 9 \mathrm{THz}$.

To demonstrate the LMC mechanism, a large-scale underdense plasma is necessary to excite plasma waves efficiently. Almost all of the previously reported experiments on $\mathrm{THz}$ radiation from solid targets are pumped with femtosecond laser pulses. We have studied a different regime, where a relativistic picosecond laser pulse interacts with a large-scale inhomogeneous preplasma in front of solid targets [46]. The experiment was carried out using the COMET laser system at the Lawrence Livermore National Laboratory. A $1053 \mathrm{~nm} 0.5$ ps laser pulse was focused onto a $1 \mathrm{~mm}$ thick copper target at an incidence angle of $62.5^{\circ}$. The peak irradiance was $\sim 5 \times 10^{17} \mathrm{~W} / \mathrm{cm}^{2}$ for $1 \mathrm{~J}$ laser energy. To generate a controllable quasi-one-dimensional large-scale preplasma, a $1053 \mathrm{~nm}, 0.5 \mathrm{ps}$ prepulse was incident onto the target with a focal diameter of $\sim 100 \mu \mathrm{m}$ in advance of the main laser pulse. The preplasma scalelength was adjusted by varying the energy or the timing of the prepulse, and measured by a Nomarski interferometer. The $\mathrm{THz}$ radiation was detected with a pyroelectric detector in the specular $\left(62.5^{\circ}\right)$ direction. 

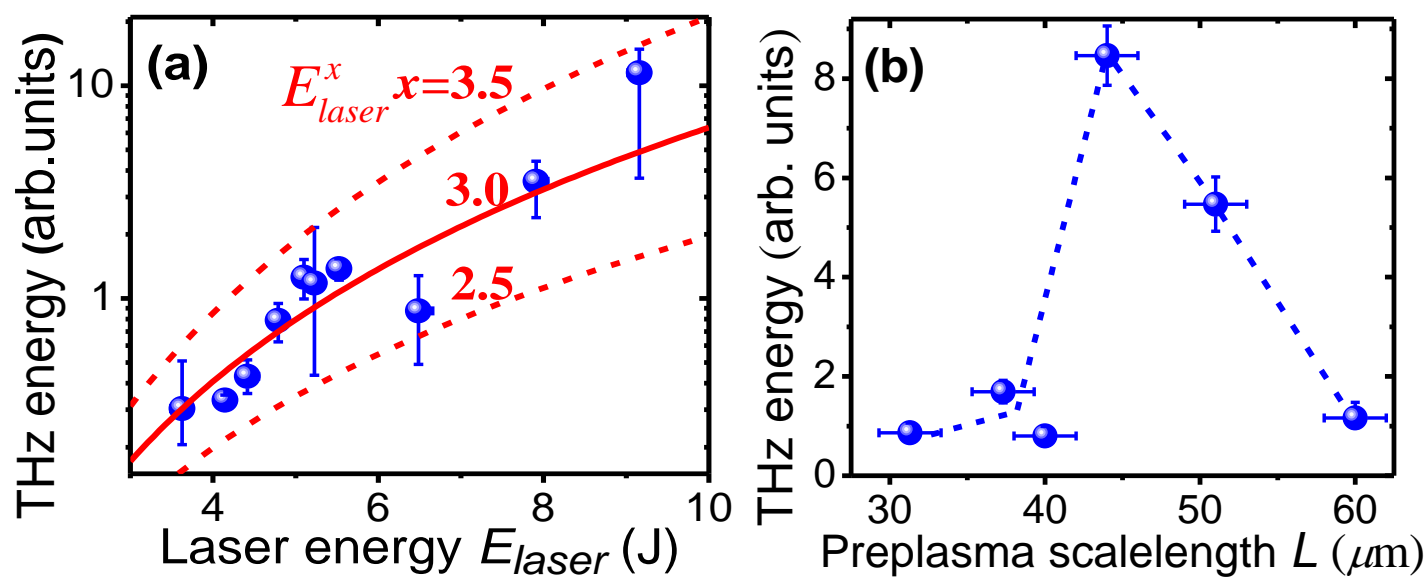

Figure 5. THz energy as a function of (a) laser energy and (b) preplasma scalelength L. From Liao et al 2015 [46].

Figure 5(a) shows the $\mathrm{THz}$ radiation as a function of the laser energy. The $\mathrm{THz}$ radiation increases nonlinearly as a power scaling with an exponent of $\sim 3 \pm 0.5$ as the laser energy is increased. No trend of saturation of the radiation is observed. Figure 5(b) shows the measured dependence of THz radiation on the preplasma scalelengths. One can see there is an optimal scalelength near $45 \mu \mathrm{m}$. Besides, spectral and polarization measurements show that the $\mathrm{THz}$ radiation is dominated by highfrequency (>10 THz) components and behaves like a $p$-polarized pulse.

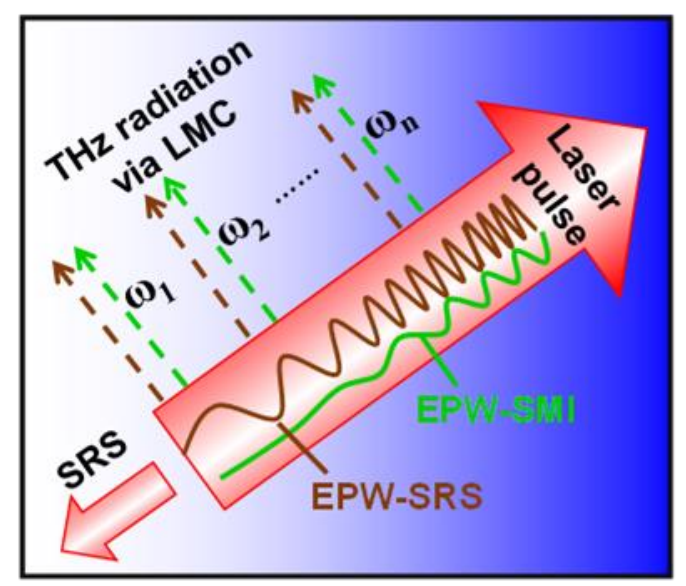

Figure 6. Schematic diagram showing the generation of THz radiation via LMC in an inhomogeneous plasma.

From Liao et al 2015 [46].

There are two ways to excite large-amplitude EPWs for a long laser pulse in a large-scale plasma, as shown in figure 6. One is stimulated Raman scattering (SRS), which occurs spontaneously during the laser propagation in a large-scale plasma [43]. The other is relativistic self-modulated laser wakefield excitation, which occurs as a result of relativistic self-modulation instability (SMI) [47]. In 
inhomogeneous plasmas, the plasma waves excited by SRS and SMI could be partially converted into electromagnetic waves via mode conversion. Since the plasma density in front of solid targets is distributed over a very large range, the mode-converted $\mathrm{THz}$ radiation would be ultra-broadband with high frequency components. Our PIC simulations have demonstrated those processes, and reproduced the observed optimal plasma scalelength [46]. Based on the LMC model, the THz radiation energy should scale with the square of the laser intensity in the weakly relativistic approximation [45]. While for the relativistic laser regime investigated in the present experiment, the plasma wave amplitude will increase nonlinearly with the laser intensity. This will result in a power exponent higher than 2 , which agrees with the measured exponent of $\sim 3 \pm 0.5$, as shown in figure 5 (a).

\section{Summary}

Our recent studies of the $\mathrm{THz}$ radiation generated from relativistic laser-solid interactions have been reviewed. The $\mathrm{THz}$ radiation properties and the underlying generation mechanisms are found to be critically dependent on the laser-plasma conditions. The $\mathrm{THz}$ radiation from the rear of a foil target is attributed to the coherent transition radiation excited by the fast electrons crossing the targetvacuum boundary in our experiments. The target surface transient current is responsible for the $\mathrm{THz}$ radiation from the front of a solid target with a relatively small preplasma. A plasma density scalelength less than several micrometers is necessary to enhance the low-frequency $\mathrm{THz}$ radiation generated by the SFE beam. While for the case where a laser pulse interacts with a large-scale plasma of a density scalelength of tens micrometers, electrostatic-electromagnetic mode conversion could play an important role in the THz generation. The understanding of those generation mechanisms will help to enhance our control of such laser-plasma-based THz sources. These sources with high peak power can be applied to the study of nonlinear interactions of high-field THz waves with matter.

\section{Acknowledgments}

We wish to acknowledge the laser facility team at SJTU, LLNL and INRS-EMT for laser operation and technical support. This work is supported by the National Basic Research Program of China (Grants No. 2013CBA01501 and No. 2014CB339801), the National Nature Science Foundation of China (Grants No. 11520101003, No. 11135012, No. 11375262, and No. 11421064). The work of one author (J.N.) was performed under the auspices of the U.S. Department of Energy 
by Lawrence Livermore National Laboratory under Contract DE-AC52-07NA27344.

\section{References}

[1] Kampfrath T, Tanaka K and Nelson K A 2013 Nat. Photon. 7680

[2] Pickwell E and Wallace V P 2006 J. Phys. D: Appl. Phys. 39 R301

[3] Nagatsuma T, Ducournau G and Renaud C C 2016 Nat. Photon. 10371

[4] Ropagnol X, Blanchard F, Ozaki T and Reid M 2013 Appl. Phys. Lett. 103161108

[5] Hebling J, Almási G, Kozma I Z and Kuhl J 2002 Opt. Express 101161

[6] Shalaby M and Hauri C P 2015 Nat. Commun. 65976

[7] D’Amico C et al 2007 Phys. Rev. Lett. 98235002

[8] Cook D J and Hochstrasser R M 2000 Opt. Lett. 251210

[9] Li Y T, Wang W M, Li C and Sheng Z M 2012 Chin Phys B 21095203

[10] Houard A et al 2008 Phys. Rev. Lett. 100255006

[11] Xie X, Dai J and Zhang X C 2006 Phys. Rev. Lett. 96075005

[12] Clerici M et al 2013 Phys. Rev. Lett. 110253901

[13] Kim K Y, Taylor A J, Glownia J H and Rodriguez G 2008 Nat. Photon. 2605

[14] Leemans W P et al 2003 Phys. Rev. Lett. 91074802

[15] Hamster H et al 1993 Phys. Rev. Lett. 712725

[16] Sagisaka A et al 2008 Appl. Phys. B 90373

[17] Gao Y, Drake T, Chen Z and DeCamp M F 2008 Opt. Lett. 332776

[18] Tokita S et al 2015 Sci. Rep. 58268

[19] Poyé A et al 2015 Phys. Rev. E 91043106

[20] Quinn K et al 2009 Phys. Rev. Lett. 102194801

[21] Gopal A et al 2013 Opt. Lett. 384705

[22] Gopal A et al 2013 Phys. Rev. Lett. 111074802

[23] Ding W J, Sheng Z M and Koh W S 2013 Appl. Phys. Lett. 103204107

[24] Liao G Q et al 2016 Phys. Rev. Lett. 116205003

[25] Schroeder C B, Esarey E, Tilborg J van and Leemans W P 2004 Phys. Rev. E 69016501

[26] Popescu H et al 2005 Phys. Plasmas 12063106

[27] Lee Y S 2009 Principles of Terahertz Science and Technology (Springer)

[28] Wharton K B et al 1998 Phys. Rev. Lett. 81822

[29] Chen L M et al 2001 Phys. Rev. E 63036403

[30] Lepore J V and Riddell Jr R J 1976 Phys. Rev. D 132300 
[31] Yuan L C L, Wang C L, Uto H and Prünster S 1970 Phys. Rev. Lett. 251513

[32] Wu Z et al 2013 Rev. Sci. Instrum. 84022701

[33] Li C et al 2016 Opt. Express 244010

[34] Li Y T et al 2006 Phys. Rev. Lett. 96165003

[35] Chen M et al 2006 Opt. Express 143093

[36] Reich Ch et al 2003 Phys. Rev. E 68056408

[37] Jackson J D 1999 Classical Electrodynamics (Wiley: New York, 3rd ed.)

[38] Liao G Q et al 2016 Phys. Plasmas 23013104

[39] Li Y T et al 2012 Appl. Phys. Lett. 100254101

[40] Li C et al 2011 Phys. Rev. E 84036405

[41] Yuan X H et al 2014 High Power Laser Sci. Eng. 25

[42] Li C et al 2014 Opt. Express 2211797

[43] Kruer W L 2003 The Physics of Laser Plasma Interactions (Addison-Wesley: New York)

[44] Hinkel-Lipsker D E, Fried B D and Morales G J 1989 Phys. Rev. Lett. 622680

[45] Sheng Z M, Mima K, Zhang J and Sanuki H 2005 Phys. Rev. Lett. 94095003

[46] Liao G Q et al 2015 Phys. Rev. Lett. 114255001

[47] Krall J, Ting A, Esarey E and Sprangle P 1993 Phys. Rev. E 482157 